\title{
Capacitação profissional do enfermeiro de um complexo hospitalar de ensino na visão de seus gestores*
}

\author{
Directors of nursing point of view of the professional capacitating program for nurses working in \\ major teaching medical centers \\ Capacitación profesional del enfermero de un complejo hospitalario de enseñanza en la visión de \\ sus gestores
}

Juliana Caires de Oliveira Achili Ferreira ${ }^{1}$, Paulina Kurcgant ${ }^{2}$

\begin{abstract}
RESUMO
Objetivo: Caracterizar o perfil de capacitação profissional do enfermeiro de um Complexo Hospitalar de Ensino, a partir da percepção das Diretoras de Enfermagem. Métodos: Nesta pesquisa qualitativa utilizou-se o método do Estudo de Caso e adotou-se a técnica da entrevista semi-estruturada realizada com 12 colaboradores. Resultados: Os dados coletados, analisados utilizando-se a Análise de Conteúdo, possibilitaram o resgate das Categorias Política Institucional, Política de Gerenciamento de Recursos Humanos e Elementos Intervenientes no Processo de Capacitação Profissional do Enfermeiro. Conclusões: Não há uma política institucional formalizada de capacitação do enfermeiro; a questão salarial é elemento dificultador para a capacitação; a contrapartida das instituições de ensino aparece como recurso para capacitação; dois Institutos não possuem Serviço de Educação Continuada e o perfil demandado é de um profissional com postura ética, conhecimento técnico-científico, capacidade de gerenciar sua equipe, assíduo, pontual e comprometido com a Instituição.

Descritores: Capacitação de recursos humanos em saúde; Recursos humanos de enfermagem/educação; Capacitação profissional; Educação continuada em enfermagem
\end{abstract}

\begin{abstract}
Objective: To characterize the components of a capacitating program for nurses working in major teaching medical centers. Methods: A case study approach was used to conduct this study. Data were collected through semi-structured interviews with 12 directors of nursing. Data were analyzed through content analysis. Results: The findings consisted of the following themes: Institutional politics, human resources policies, and specific intervening elements of the professional capacitating program for nurses. Conclusions: There were no formal institutional policies for professional capacitation of nurses. The nurses' salary level was an element that made the implementation of a professional capacitating program for nurses difficult. Two medical centers did not have a department of continuing education. In addition, there was a need for capacitating nurses to: behave ethically; develop scientific, technical, and management knowledge; and, be assiduous, punctual, and committed to the institution.
\end{abstract}

Keywords: Health human resource training; Nursing staff/education; Professional training; Education, nursing, continuing

\section{RESUMEN}

Objetivo: Caracterizar el perfil de capacitación profesional del enfermero de un Complejo Hospitalario de Enseñanza, a partir de la percepción de las Directoras de Enfermería. Métodos: en esta investigación cualitativa se utilizó el método del Estudio de Caso adoptándose la técnica de la entrevista semi-estructurada realizada con 12 colaboradores. Resultados: Los datos recolectados, analizados por medio del Análisis de Contenido, posibilitaron el rescate de las Categorías Política Institucional, Política de Gerenciamiento de Recursos Humanos y Elementos Intervinientes en el Proceso de Capacitación Profesional del Enfermero. Conclusiones: No hay una política institucional formalizada de capacitación del enfermero; la cuestión salarial es el elemento que dificulta la capacitación; la contrapartida de las instituciones de enseñanza aparece como recurso para capacitación; dos Institutos no poseen Servicio de Educación Continua y el perfil demandado es el de un profesional con postura ética, conocimiento técnico-científico, capacidad para gerenciar su equipo, asiduo, puntual y comprometido con la Institución.

Descriptores: Capacitación de recursos humanos en salud; Personal de enfermería/educación; Capacitación profesional; Educación continua en enfermería

* Trabalbo extraído da Tese de Mestrado Perfil de capacitação profissional do enfermeiro de um Complexo Hospitalar de Ensino, defendida na Escola de Enfermagem da Universidade de São Paulo - USP - São Paulo (SP), Brasil.

${ }^{1}$ Enfermeira, Mestre em Administração de Serviços de Enfermagem da Escola de Enfermagem da Universidade de São Paulo - USP - São Paulo (SP), Brasil.

${ }^{2}$ Doutora, Professora Titular do Departamento de Orientação Profissional da Escola de Enfermagem da Universidade de São Paulo - USP - São Paulo (SP), Brasil. 


\section{INTRODUÇÃO}

O enfermeiro está presente em todos os níveis de prestação de serviços à saúde, desde a atenção básica até os serviços da mais alta complexidade; bem como atua no ensino do pessoal de nível médio, técnico e nos cursos de graduação e pós-graduação em enfermagem. Também tem divulgado sua produção técnico-científica, contribuindo para elucidar a transformação dos processos assistenciais e para a construção de novos conhecimentos sobre o cuidado de enfermagem em saúde $^{(1)}$.

Atualmente, pela análise do mercado de trabalho e das diversas áreas de atuação do enfermeiro, percebe-se, a competência profissional como requisito básico, uma vez que se vive a era da informação e do conhecimento, buscando-se padrões de excelência, alcançando-se melhores resultados organizacionais e aumentando a competitividade. A noção de competência possibilita o desenvolvimento de enfermeiros pensantes, capazes de reflexão/ação social crítica e de opção pela postura de sujeitos construtores do conhecimento na, com a, e para a, prática profissional da enfermagem, nos diversos âmbitos e lugares sociais em que se realiza ${ }^{(2)}$.

Dessa forma, é relevante o fato de que o enfermeiro deve ter iniciativa e disponibilidade para desenvolver sua competência, e que Complexos Hospitalares de Hospitais de Ensino, em geral, albergam significativo número de enfermeiros locados nos diferentes Institutos. Cada Instituto guarda sua especificidade técnica, assistencial e gerencial, e o aprofundamento da capacitação profissional, na maioria das vezes, ocorre concomitantemente ao trabalho, dependendo, portanto, de concessões da Instituição, motivação e disponibilidade pessoal, salientando-se, assim, as diferenças do comportamento humano na organização hospitalar.

Nesse âmbito, a educação continuada é considerada também fundamental para uma tomada de consciência da necessidade da formação profissional sistemática, programada de modo a atingir todos os níveis, oferecendo a todos a oportunidade de crescimento profissional e pessoal. Assim, o enfermeiro é um educador em qualquer campo de atuação, seja no ensino ou na assistência, sendo este processo de trabalho tão importante quanto o de pesquisa ou o administrativo ${ }^{(3)}$. A eficácia do treinamento de enfermeiros para uma assistência à saúde, em nível hospitalar, depende diretamente das experiências de ensinoaprendizagem proporcionadas por essa Instituição, cujo programa global deve conter as ações de saúde que lhe compete prestar à população que lhe é adstrita. Isto significa que é intrínseco ao hospital, como a qualquer instituição produtora de bens ou serviços, responsabilizarse pelo contínuo aperfeiçoamento de todo o seu corpo de pessoal ${ }^{(4)}$.
$\mathrm{Na}$ enfermagem, essa responsabilidade de treinamento e conseqüente capacitação está diretamente ligada ao serviço de Educação Continuada, que é o órgão co-responsável por treinar e capacitar os funcionários, a fim de realizarem adequadamente suas atribuições. Para tanto, é necessário o planejamento detalhado das propostas de capacitação de pessoal que alberguem conhecimento nas dimensões técnicocientífica, ético-política e sócio-educativa. É necessário, ainda, que envolvam todas as dimensões do ser humano, ressaltando os valores sociais, políticos, religiosos e filosóficos que influenciam a percepção, o raciocínio, o julgamento e as decisões do aprendiz.

Assim, os gestores e as instituições hospitalares devem investir na capacitação dos recursos humanos, considerando os contextos sócio-político-econômico e de saúde vigentes. Nessa direção, o gerente de recursos humanos de enfermagem, assim como os demais gerentes em saúde devem proporcionar condições que garantam a efetiva contribuição dos profissionais e trabalhadores na obtenção dos objetivos institucionais, com o estabelecimento de relações de colaboração entre os trabalhadores, os usuários, e as instituições e suas respectivas gerências ${ }^{(5)}$.

Com esta leitura da realidade, a presente pesquisa teve como objetivo geral: Caracterizar um perfil de capacitação profissional do enfermeiro a partir da percepção das Diretoras de Divisão de Enfermagem dos Institutos que compõem o Complexo Hospitalar do Hospital das Clínicas da Faculdade de Medicina da Universidade de São Paulo. Como objetivos específicos: Identificar a percepção das diretoras da Divisão de Enfermagem acerca do perfil de capacitação dos enfermeiros; Conhecer as propostas que orientam essa capacitação; e Analisar os elementos institucionais necessários para a efetivação desse processo.

\section{MÉTODOS}

Esta pesquisa, de caráter descritivo-exploratório, foi conduzida pela abordagem qualitativa e desenvolvida no pólo central do Complexo Hospitalar do Hospital das Clínicas da Faculdade de Medicina da Universidade de São Paulo, após aprovação do Comitê de Ética em Pesquisa da Escola de Enfermagem da Universidade de São Paulo e da Comissão de Ética para Análise de Projetos de Pesquisa do Hospital das Clínicas da Faculdade de Medicina da Universidade de São Paulo. Participaram da pesquisa as seis Diretoras de Divisão de Enfermagem dos Institutos e suas substitutas, totalizando 12 participantes, que assinaram o Termo de Consentimento Livre e Esclarecido.

O método adotado foi o Estudo de Caso e para coleta de dados foi utilizada a técnica da entrevista semiestruturada. A caracterização das participantes deu-se a partir de questões referentes ao cargo e à trajetória 
institucional, enquanto a entrevista semi-estruturada teve por base três questões norteadoras: Como você percebe o processo de capacitação profissional do enfermeiro neste Instituto? O que você propõe e considera importante nessa capacitação? Que elementos você considera necessários para a efetivação desse processo?

Para análise dos dados obtidos nas entrevistas, adotouse a técnica da Análise de Conteúdo, definida por Bardin ${ }^{(6)}$ como um conjunto de técnicas de análise de comunicação que visa obter, através da descrição do conteúdo das mensagens, indicadores que permitem a inferência de conhecimentos relativos às condições de produção e recepção destas mensagens. A autora assinala três etapas básicas para a análise, e as denomina de pólos cronológicos: pré-análise: fase de organização, objetiva sistematizar idéias iniciais, escolha dos documentos, formulação das hipóteses e dos objetivos e elaboração de indicadores que fundamentem a interpretação final; exploração do material: fase longa e fastidiosa, que consiste em operações de codificação, transformando os dados brutos do texto e permitindo atingir uma representação do conteúdo; tratamento dos resultados com a inferência e a interpretação.

\section{RESULTADOS}

Quanto à caracterização das colaboradoras deste estudo, a idade variou de 28 a 70 anos, e o tempo de trabalho na instituição de 6 a 38 anos, revelando uma diferença significativa entre elas. $\mathrm{O}$ ano de formação na graduação, o tempo no cargo e cursos de pós-graduação também são diversificados. Chama a atenção o fato de todas as colaboradoras, em sua trajetória profissional no Hospital das Clínicas, terem trabalhado como enfermeiras assistenciais antes de assumirem o cargo que atualmente ocupam.

A análise do conteúdo das entrevistas permitiu a construção de três categorias e suas respectivas Unidades de Significado:

Categoria Política Institucional com as Unidades de Significado Diretriz Institucional, Necessidade de Mudança, Perfil Desejado e Responsabilidades do Gerente de Enfermagem;

Categoria Política de Gerenciamento de Recursos Humanos com as Unidades de Significado Capacitação Anterior, Perfil atual, Critérios para Capacitação, Processo Admissional, Critérios Seletivos e Diretrizes para Capacitação do Pessoal de Enfermagem;

Categoria Elementos intervenientes no processo de capacitação profissional do enfermeiro com as Unidades de Significado Fatores Facilitadores, Fatores Dificultadores e Fatores Facilitadores e Dificultadores.

Para a compreensão da realidade organizacional, é necessário captar os elementos constitutivos da estrutura formal e informal e analisá-los de forma conjunta, assim como os padrões culturais específicos, os processos e as relações de poder que determinam a dinâmica organizacional, sendo importante enfatizar que, nas organizações, quando os funcionários traduzem seu poder em ações, estão fazendo política ${ }^{(7-8)}$.

Nesta pesquisa, embora não de forma documentada, a política institucional permeia todo o processo de capacitação de pessoal. Assim, as entrevistadas, em seus discursos, explicitaram a existência de elementos do processo de trabalho constitutivos e concretizadores da política organizacional, manifestando a necessidade e a importância da formalização dessa política.

Nesse contexto, os resultados do estudo mostraram que existem semelhanças nas falas referentes ao processo admissional, evidenciando que a admissão de enfermeiros recém-formados por concurso público é um fator dificultador de capacitação profissional, pois muitos profissionais chegam sem os conhecimentos básicos de enfermagem, o que dificulta a dinâmica admissional, conforme mostra a fala:

Muitos enfermeiros chegam aqui recém-formados, não têm experiência, aquela bagagem de cuidado, ele vem cru, não tem babilidade... (E1D1)

Nessa vertente, 11 colaboradoras trouxeram, em seus discursos, a necessidade de se estabelecer um perfil do profissional demandado, que mesmo não formalizado, é um elo comum na concepção das colaboradoras, dos diferentes Institutos, quando demandam um profissional com postura ética, conhecimento técnico-científico, capacidade de gerenciar sua equipe, assíduo, pontual e comprometido com a Instituição. Outros elementos demandados por elas, referentes ao perfil profissional, foram considerados de forma diferenciada, como a questão da especialização e da experiência profissional, do relacionamento e do comportamento profissional.

A análise dos dados também mostrou que existe preocupação por parte de todas quanto à capacitação profissional. Entretanto, nos manuais que tratam das atividades desenvolvidas pelos enfermeiros, não há referências a respeito das competências necessárias para o desenvolvimento dessas atividades.

Verificou-se que não existe política formalizada de capacitação do enfermeiro no Complexo Hospitalar em estudo, bem como nos Institutos, separadamente. Desta forma, cada Instituto trabalha a capacitação do enfermeiro de maneira particular e as colaboradoras desta pesquisa têm, em suas mãos, a responsabilidade de efetivar esta formalização. Este fato é evidenciado nas falas seqüenciais:

Não existe uma politica estruturada, os diretores de serviço vêem a necessidade de seu grupo e atuam ali. (E1D3)

Não tem uma política formalizada, então fazemos o planejamento 
contendo o que consideramos importante para o desenvolvimento da função deste profissional. (E4D4)

Assim como o perfil desejado, todas revelaram seguir diretrizes semelhantes para capacitação do pessoal de enfermagem, que vão desde o estímulo para capacitação externa, devido a verbas insuficientes, até a capacitação durante horário de trabalho. Também foi revelado que há preocupação com a capacitação dos enfermeiros chefes e supervisores; com a programação anual de capacitação dos enfermeiros; com as oportunidades para os profissionais realizarem aprimoramento nos próprios Institutos; com a capacitação profissional fora do horário de trabalho; com a preferência por contratar exaprimorandos e com o incentivo para essa capacitação.

Entre os elementos considerados necessários para que a capacitação profissional aconteça, está o Serviço de Educação Continuada, que ainda não é uma realidade em todos os Institutos pesquisados devido, principalmente, à falta de recursos humanos como uma questão decorrente de política de recursos humanos, ressaltando que o quadro de enfermeiros aprovado pelo Governo do Estado data de 1978. A fala a seguir mostra:

Atualmente não existe aqui o Serviço de Educaşão Continuada, é uma proposta, estamos tentando criar o serviço... Teria que ter um profissional, mas pelo quadro reduzido de enfermeiros não temos um profissional que possa assumir este posto. (E4D5)

Ainda referente aos elementos necessários para a capacitação, emergem das entrevistas a inexistência de um manual de capacitação profissional e a falta de recursos físicos, financeiros e humanos para a efetivação dessa capacitação. As colaboradoras revelam que a questão salarial aparece como um impedimento para manter o corpo de enfermeiros capacitados, pelo fato do salário estar aquém do existente no mercado. Este fato faz com que a rotatividade esteja presente nos Institutos, evidenciando um problema comum a todo o Complexo Hospitalar e mostrando que o Hospital das Clínicas (HC) tem, entre suas características, o fato de ser um órgão formador de profissionais para o mercado. Assim, além de hospital escola, o HC trabalha com programas de residência, especialização e aprimoramento, recebendo profissionais através de concurso público que, após um ou dois anos de trabalho adquirem experiência e deixam o hospital por ofertas melhores nos hospitais privados, como mostra a fala:

Vários profissionais que foram especialistas daqui da casa, estão todos trabalhando em hospitais que a gente conhece, e a gente não os recupera pela questão salarial, porque está pesando muito hoje em dia a questão salarial. (E3D3)

Por outro lado, o número de enfermeiros especialistas lotados nos Institutos é significativo e a busca dos profissionais por mestrado e doutorado vem crescendo. O campo para desenvolvimento de pesquisas é vasto e a conscientização e preocupação em escrever, pesquisar, produzir, foi evidenciada nas falas das diretoras e das enfermeiras substitutas, como passo importante na trajetória de capacitação que vem ocorrendo.

A contrapartida das instituições de ensino que buscam campo de estágio no Complexo Hospitalar é revelada nos discursos das colaboradoras como um recurso para atender às necessidades de capacitação do enfermeiro, em especial pelo fato de ser um hospital público, cuja verba para essa capacitação é pequena e não atende à demanda, principalmente da enfermagem. Desta forma, a contrapartida das instituições de ensino pode ser considerada um elemento que, atualmente, integra a política de capacitação de recursos humanos deste Complexo Hospitalar. A fala a seguir reflete essa realidade:

Este ano, para aceitar estágio curricular aqui na Instituição, tenho que negociar com a escola uma contra-partida. Então se vão mandar alguns alunos pra cá, eu vou ver qual dos cursos que interessa pra nós que algumas das enfermeiras façam e elas também têm interesse. Isso vai valer pra todas as escolas, é uma medida, é um novo pensamento da Superintendência, do Núcleo de Capacitação e Desenvolvimento. (E5D3)

Por fim, outro aspecto levantado pelas colaboradoras do estudo diz respeito ao processo seletivo e admissional do enfermeiro, que também difere entre os institutos, ressaltando que um destes Institutos ainda não tem quadro de pessoal aprovado pelo Governo do Estado. Em comum, o que existe é a obrigatoriedade de ser enfermeiro e estar legalmente habilitado para exercer a função, bem como outros atributos solicitados no edital fornecido pelo setor de recursos humanos do Complexo Hospitalar.

\section{DISCUSSÃO}

Nas organizações de saúde a estrutura formal estabelece os níveis hierárquicos dos serviços e dos agentes sociais, indicando, pelas diferentes posições no desenho organizacional, as relações de poder entre os cargos. Existem estruturas próprias que guardam semelhanças entre si quando pertencentes a um mesmo seguimento de trabalho humano, e que, junto com materiais adotados e atividades desenvolvidas, estabelecem um perfil organizacional próprio(7).

Desta forma, o processo de capacitação profissional do enfermeiro no ambiente hospitalar em estudo está inserido no contexto de uma estrutura organizacional complexa, necessitando, assim, de uma estrutura sólida para ser desenvolvido adequadamente. 
Estudiosos de Gerenciamento de Recursos Humanos consideram que os responsáveis pelo recrutamento de pessoas enfrentam grande dificuldade em conseguir, no mercado de trabalho, profissionais habilitados para ocuparem as vagas disponíveis, o que coloca em discussão a eficiência do processo de formação e qualificação desses profissionais ${ }^{(9)}$. As falas das colaboradoras desta pesquisa vêm ao encontro dessa problemática, trazendo a falta de conhecimento e experiência como um fator que prejudica o desempenho do profissional, pelo fato da graduação não dar um enfoque maior nas especialidades, fazendo com que os profissionais recémformados saiam da graduação sem conhecimento técnicocientífico e prático adequados.

O perfil deve ser ajustado às necessidades do trabalho e do indivíduo, e a organização deve elaborar critérios de seleção com a finalidade de propiciar comparação entre o perfil estabelecido e o perfil do candidato, visando o alcance dos resultados esperados e a integração do novo profissional ao grupo de trabalho ${ }^{(7)}$. Nesta pesquisa, no que se refere ao perfil desejado, foram encontrados os elementos especialização, conhecimento técnico-científico, experiência profissional, comprometimento com a Instituição e o gerenciamento, que compõem o perfil desejado, além de assiduidade, postura, pontualidade e ética.

A duração, o conteúdo, os métodos adotados, a avaliação dos resultados, as estratégias para a integração do recém-admitido, suas experiências de trabalho e as relações com os profissionais do setor são aspectos fundamentais na elaboração e operacionalização dos programas de capacitação. Todavia, há poucos profissionais de educação continuada nas instituições, dificultando o acompanhamento individualizado do recém-admitido e sua avaliação, confirmando que as instituições necessitam investir em seus recursos humanos para contar com pessoal eficiente e qualificado, mas é necessário que os profissionais também tomem iniciativas, buscando meios e formas para sua própria qualificação ${ }^{(10-11)}$.

O mercado de trabalho solicita, cada vez mais, que um profissional tenha conhecimentos e domine técnicas, o que contribui para que muitos estudantes decidam fazer pós-graduação com o intuito de ampliar sua competência, ou seja, o curso de graduação não é suficiente para que os recém-formados possam atender à demanda do perfil profissional solicitado pelo mercado de trabalho ${ }^{(9)}$.

Estudo realizado em $1996^{(12)}$ já tecia considerações sobre este tema, revelando a necessidade de se aliar os cursos de graduação às instituições hospitalares. As autoras consideram que este fato favorece o desenvolvimento da assistência e, conseqüentemente, da profissão, enfatizando um

\section{REFERÊNCIAS}

1. Santos $\mathrm{CH}$, Mendes TR. Competência e autonomia do enfermeiro na contemporaneidade. REME Rev Min Enferm. 2001;5(1/2):101-2. aprendizado gerencial através de um programa permanente de formação de recursos humanos no hospital, e a abordagem, nos curso de graduação de temas referentes ao processo decisório do enfermeiro. Esses temas devem privilegiar a gerência como fator determinante para atingir o paciente e os objetivos organizacionais, mediante o planejamento, a coordenação, e a avaliação da assistência prestada.

\section{CONCLUSÃO}

Nos dias de hoje, é inquestionável para a maioria das organizações a necessidade de capacitar seus profissionais, por meio de uma educação reflexiva e participativa, impulsionada por pressões sociais, como elevação da escolaridade, crescente aumento do nível de informação das pessoas e inovações tecnológicas, bem como motivação e expectativa das pessoas para participação nas decisões, nos resultados e no futuro da empresa. Com o surgimento dos hospitais e das clínicas e com a incorporação de novas tecnologias, novas exigências foram feitas em termos de administração hospitalar e de pessoal para a assistência de enfermagem, assim como novos núcleos de preparação de enfermeiros em diferentes Estados da Federação Brasileira, além do serviço de enfermagem ter, entre as atribuições, desenvolver programa de educação em serviço a fim de garantir a utilização máxima das habilidades e potencial de sua equipe de trabalho, capacitando os próprios recursos humanos ${ }^{(7,13)}$.

O presente estudo buscou caracterizar o perfil de capacitação profissional do enfermeiro de um Complexo Hospitalar de Ensino, a partir da visão de seus gestores.

Através da análise dos dados foi possível perceber que, apesar de não haver uma política formalizada de capacitação do enfermeiro, existe o empenho dos enfermeiros e das chefias de enfermagem para o desenvolvimento dessa capacitação profissional.

A Coordenação das Atividades de Enfermagem (CAENF), composta pelas Diretoras de Divisão de Enfermagem dos Institutos, possui regimento, é institucionalizada, realiza reuniões mensais que tratam de assuntos referentes à enfermagem do Complexo Hospitalar estudado, e promove, a cada dois anos, um Congresso de Enfermeiros, coordenado por um dos Institutos. A CAENF tem, entre os objetivos para o futuro, a proposta de montar um grupo/recurso para a capacitação e desenvolvimento do pessoal. Entretanto, essa disposição não tem sido elemento suficiente para superar as dificuldades de ordem política e econômica que perpassam um Complexo Hospitalar Público.

2. Gabrielli JMW. Formação do enfermeiro: buracos negros e pontos de luz. [tese]. Ribeirão Preto: Escola de Enfermagem de Ribeirão Preto da Universidade de São Paulo; 2004. 
3. Padilha MICS. A prática da educação em serviço na instituição privada. Enfoque (São Paulo). 1991;19(3):55-8.

4. Carvalho JF, Paim L. O papel do hospital no treinamento de enfermeiros para a assistência primária de saúde. Rev Bras Enferm. 1981;34(1):35-40.

5. Peduzzi M. Política de recursos humanos nas organizações de saúde. São Paulo: Escola de Enfermagem da USP; 2000.

6. Bardin L. Análise de conteúdo. Lisboa: Edições 70; 1977.

7. Kurcgant P, coordenadora. Gerenciamento em enfermagem. Rio de Janeiro: Guanabara-Koogan; 2005.

8. Robbins SP. Comportamento organizacional. Trad. de Reynaldo Marcondes. São Paulo: Pearson Prentice Hall; 2005.

9. Gondim SMG. Perfil profissional e mercado de trabalho: relação com a formação acadêmica pela perspectiva de estudantes universitários. Est Psicol. 2002;7(2):299-309.

10. Siqueira ILCP, Kurcgant P. Estratégia de capacitação de enfermeiros recém-admitidos em unidades de internação geral. Rev Esc Enferm USP. 2005; 39(3):251-7.

11. Oguisso T. A Educação continuada como fator de mudanças: visão mundial. Nursing (São Paulo). 2000;3(20):22-9.

12. Bocchi SCM, Fávero N. Caracterização das atividades diárias do enfermeiro chefe de seção em um hospital universitário. Rev Latinoam Enferm. 1996;4(2):41-59.

13. Programa de Estudos Avançados em Administração Hospitalar e de Sistemas de Saúde (PROHASA). Manual de organização e procedimentos hospitalares. São Paulo: FGV/EAESP/HC/FMUSP; 1987. 